\title{
Actividad biocida del aceite esencial de Lippia origanoides H.B.K. (Verbenaceae) sobre Rhizoctonia solani: in vitro
}

\section{Biocidal activity of Lippia origanoides H.B.K. (Verbenaceae) essential oil on Rhizoctonia solani: in vitro}
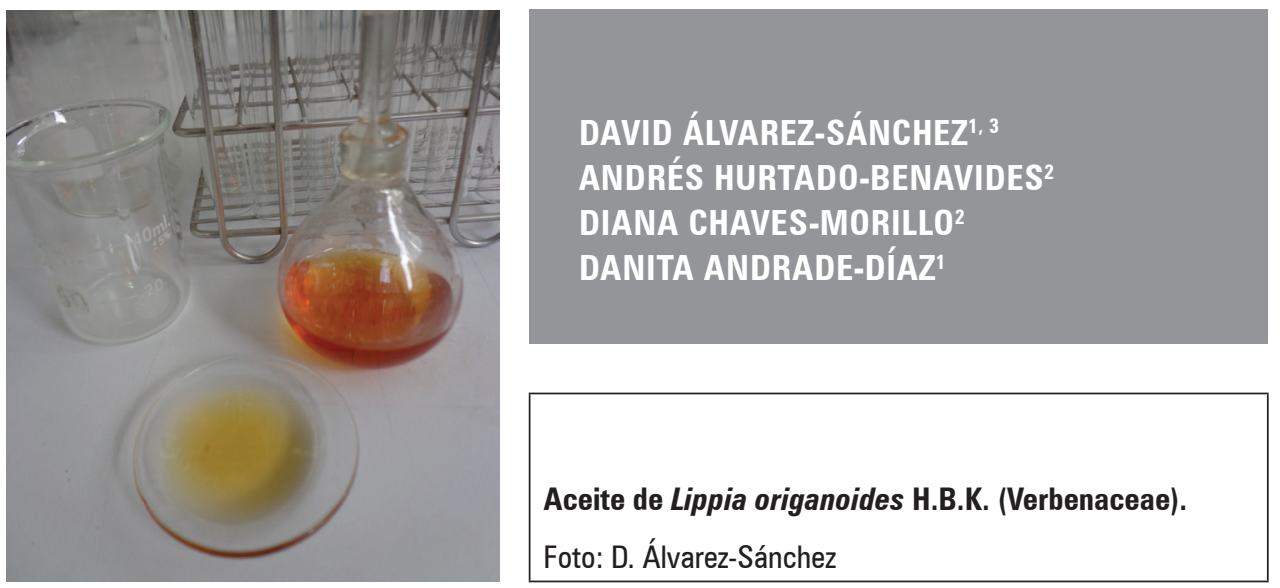

\section{RESUMEN}

Rhizoctonia solani es un hongo que afecta la producción de tubérculos y vigor de las plantas de papa Solanum tuberosum L. Las consecuencias ambientales que genera su control han impulsado la búsqueda de alternativas menos contaminantes, en donde los aceites esenciales tienen gran potencial. El arbusto silvestre Lippia origanoides H.B.K. (Verbenaceae) ha sido estudiado por sus propiedades fungicidas en su extracto esencial, por ello, el objetivo de esta investigación fue evaluar la actividad biocida del aceite esencial de L. origanoides sobre $R$. solani en condiciones in vitro. La extracción del aceite se realizó mediante arrastre con vapor. La composición química se determinó por cromatografía de gases-espectrometría de masas (GC-MS) y la actividad biocida del aceite se evaluó mediante la técnica de dilución en medio de cultivo, complementando los resultados con la técnica de bioautografía, la cual permitió identificar al timol como el compuesto mayoritario del aceite $(60,7 \%)$ y responsable de la inhibición del hongo. La concentración $110 \mu \mathrm{L} \mathrm{mL}^{-1}$ limitó el crecimiento de $R$. solani al afectar sus estructuras, con ello se demostró el efecto biocida del aceite esencial y el potencial agente antifúngico natural.

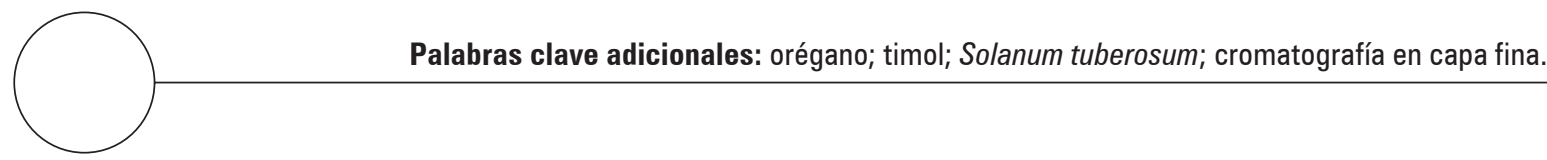

Universidad de Nariño, Facultad de Ciencias Agrícolas, Pasto (Colombia). ORCID Álvarez-Sánchez, D.: 0000-00033563-2529; ORCID Andrade-Díaz, D.: 0000-0001-7579-4739

2 Universidad de Nariño, Facultad de Ingeniería Agroindustrial, Pasto (Colombia). ORCID Hurtado-Benavides, A.: 00000002-8898-8804; ORCID Chaves-Morillo, D.: 0000-0002-5524-274X

3 Autor para correspondencia. daealvarezsa@unal.edu.co 


\section{ABSTRACT}

Rhizoctonia solani is a fungus that affects the production of tubers and vigor of potato plants, Solanum tuberosum $\mathrm{L}$. The environmental consequences generated by its control have driven the search for less contaminating alternatives, and essential oils have great potential. The wild shrub Lippia origanoides H.B.K. (Verbenaceae) has been studied because of its attractive fungicidal properties in its essential extracts; therefore, the objective of this research was to evaluate the biocidal activity of $L$. origanoides essential oil on $R$. solani, in vitro. The oil extraction was carried out with steam dragging, the chemical composition was determined with Gas Chromatography-Mass Spectrometry (GC-MS), and, finally, the biocidal activity of the oil was evaluated with the dilution technique in the culture medium, which was complemented with the bioautography technique, which identified thymol as the major compound of the oil (60.7\%), responsible for the fungus inhibition. The concentration $110 \mu \mathrm{L} \mathrm{mL}^{-1}$ limited the growth of $R$. solani by affecting the structures, thereby demonstrating the biocidal effect of this essential oil and its potential antifungal agent.

Additional key words: oregano; thymol; Solanum tuberosum; thin-layer chromatography.

Fecha de recepción: 06-03-2018 Aprobado para publicación: 30-11-2018

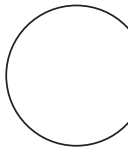

\section{INTRODUCCIÓN}

El hongo basidiomiceto Rhizoctonia solani J.G. Kühn es el fitopatógeno causante de "Rizoctoniasis", enfermedad que afecta el cultivo de papa y otras solanáceas. La infección del cultivo a menudo presenta fases iniciales de crecimiento y ejerce efectos negativos sobre el desarrollo normal de plantas y tubérculos, lo cual genera pérdidas entre el 10 y $60 \%$ del rendimiento esperado con disminución en la calidad del producto final (Medeiros et al., 2014; Kankam et al., 2016).

La diseminación y establecimiento de esta enfermedad está asociada al aumento de la humedad relativa, uso de tubérculos contaminados, escasa rotación de lotes, entre otros factores, lo cual representa un peligro latente para la mayoría de las zonas productoras, donde la aplicación de pesticidas de síntesis química se convierte en la opción más recurrente para el manejo de $R$. solani en Colombia.

Sin embargo, el uso indiscriminado de este tipo de productos puede generar externalidades negativas, como cepas del patógeno resistente a los fungicidas, contaminación ambiental, elevados costos de producción y residualidad de agroquímicos en los alimentos. Por ello, este fenómeno no solo constituye un problema ambiental sino de salud pública (Arango et al., 2014; Villa et al., 2015; Álvarez et al., 2016).

El actual interés por procesos amigables con el ambiente, agricultura sustentable y productos orgánicos, es cada vez mayor, debido a esto, la búsqueda de alternativas para el manejo de enfermedades y plagas en los cultivos ha centrado su atención en sustancias provenientes de fuentes naturales que presenten propiedades bioactivas.

En este contexto, los extractos vegetales y aceites esenciales de plantas aromáticas han sido objeto de estudio por la presencia de compuestos con actividad antimicrobiana. Adicionalmente, estos productos naturales son biodegradables y su toxicidad es baja o inexistente, por lo cual han llegado a constituirse en una opción promisoria para la sustitución de pesticidas sintéticos (Medeiros et al., 2014).

Lippia origanoides H.B.K. es una planta aromática conocida como "orégano silvestre" y en Colombia se encuentra entre los 500 y 800 msnm en ambientes secos; sus hojas han sido empleadas con fines etnobotánicos como condimento o remedio para desordenes gastrointestinales, antiséptico y tratamiento para enfermedades respiratorias (Ramírez et al., 2009; Medeiros et al., 2014; Ospina et al., 2016). De ahí, que el aceite esencial de L. origanoides ha sido estudiado por su riqueza fitoquímica, entre sus constituyentes mayoritarios se encuentran fenoles como carvacrol y timol a los cuales se les atribuye su actividad antioxidante, antifúngica y antibacterial (Arango et al., 2014).

Diferentes estudios han demostrado las propiedades biocidas de L. origanoides en la agricultura, como 
Taborda et al. (2015) que evaluaron el efecto fungicida de extractos etanólicos de L. origanoides sobre Botrytis cinerea Pers. con altos porcentajes de control. Por su parte, Arango et al. (2014) evaluaron la actividad in vitro del aceite esencial de orégano silvestre sobre Phytophthora infestans (Mont.) de Bary, obteniendo que una concentración de $150 \mu \mathrm{g} \mathrm{mL}^{-1}$ del aceite inhibió el crecimiento del patógeno. Álvarez et al. (2016) también determinaron el efecto biocida del aceite esencial sobre $P$. infestans y Alternaria solani Sorauer, en dicho estudio se evidenció el efecto del aceite sobre las estructuras reproductivas de los dos patógenos aislados de papa y se estableció que una concentración de $300 \mu \mathrm{L}$ $\mathrm{mL}^{-1}$ causa la inhibición micelial.

Los anteriores reportes bibliográficos de orégano silvestre sugieren el alto potencial en el desarrollo de tecnologías que permitan generar alternativas sostenibles para la agricultura, un campo que actualmente se encuentra en crecimiento. Teniendo en cuenta lo anterior, el objetivo del presente trabajo fue evaluar la actividad biocida del aceite esencial de L. origanoides sobre $R$. solani a nivel in vitro.

\section{MATERIALES Y MÉTODOS}

Obtención de orégano. Se colectaron hojas de Lippia origanoides H.B.K. (en floración), obtenidas de la zona del Alto Patía, vereda Alto de Mayo del municipio de Taminango (Nariño) $01^{\circ} 41^{\prime} 25,25^{\prime \prime} \mathrm{N}$ y $77^{\circ} 19^{\prime} 25,5^{\prime \prime O} \mathrm{O}$ 639 msnm y un Bosque seco tropical (bsT). El material vegetal cosechado fue dispuesto bajo sombra por un periodo de reposo de dos días $\left(24^{\circ} \mathrm{C}\right)$. Adicionalmente se envió un ejemplar con estructuras reproductivas al herbario de la Universidad de Nariño como material de referencia con el No. 45202.

Extrancción. El aceite esencial se extrajo en la planta piloto de la Universidad de Nariño en un equipo de arrastre con vapor, para este proceso se utilizó vapor saturado a presión atmosférica de 3,7 psi, densidad del lecho de $80 \mathrm{~g} \mathrm{~L}^{-1}$ con un tiempo de extracción de $2 \mathrm{~h}$ (Arango et al., 2012). La mezcla de aceite esencial-agua se separó por decantación, el volumen de aceite extraído se registró para evaluar el rendimiento.

Perfil cromatográfico del aceite esencial. Se realizó mediante cromatografía de gas acoplada a espectrometría de masas (Shimadzu GCMS-OP-20105, Kioto, Japón), según la metodología descrita por Barbosa et al. (2006) con modificaciones.
Para la separación de los componentes del aceite esencial, se utilizó la columna DB-5 de J\&W Scientific (Folsom, CA) con $30 \mathrm{~m}$ de largo, 0,25 mm de diámetro, espesor película de $0,25 \mu \mathrm{m}$, y Helio WAP como gas de arrastre. Las condiciones de preparación del cromatógrafo de gases fueron: presión interna de la columna de $56,7 \mathrm{kPa}$, relación de división 1:30, el flujo de gas en la columna 1,0 $\mathrm{mL} \mathrm{min}^{-1}$, temperatura del inyector $250^{\circ} \mathrm{C}$, temperatura del detector o interfaz (GC-MS) $280^{\circ} \mathrm{C}$. La temperatura inicial de la columna $45^{\circ} \mathrm{C}$ (5 min), seguido de un incremento de $4^{\circ} \mathrm{C} \mathrm{min}{ }^{-1}$ hasta $150^{\circ} \mathrm{C}$, y punto de equilibrio durante $30 \mathrm{~min}(2$ min), con una temperatura final de $250^{\circ} \mathrm{C}$ a razón de $5^{\circ} \mathrm{C} \mathrm{min}^{-1}$. El espectrómetro de masas fue programado para realizar lecturas en un rango de 30 a $400 \mathrm{Da}$, en intervalos de 0,5 s y energía de ionización $70 \mathrm{eV}$.

Se inyectó en el GC 1,0 $\mu \mathrm{L}$ de aceite esencial bajo una dilución al 30\% en diclorometano (HPLC). La identificación de los componentes se realizó por comparación de sus espectros de masas con los disponibles en las bases de datos de espectroteca y también por índices de Kovats (IK).

Aislamiento de $R$. solani. A partir de cultivos comerciales de papa se colectaron aislamientos de $R$. solani en medio PDA. La identificación de este microorganismo se realizó con base en las características morfológicas.

Actividad biocida. La respuesta de sensibilidad in vitro de $R$. solani se determinó mediante la técnica de dilución en medio de cultivo usada por Álvarez et al. (2016) Se incorporó aceite esencial y tween 20 en una relación 1:1 (v/v) al medio de cultivo PDA previamente esterilizado, el emulsificante se escogió debido a que estudios previos no mostraron actividad inhibitoria sobre el blanco biológico. Se sembró un disco de micelio de $1,1 \mathrm{~cm}$ de diámetro obtenido con sacabocado de aislamientos puros del fitopatógeno sembrados $8 \mathrm{~d}$ antes del ensayo.

Se establecieron tres ensayos para determinar la concentración mínima de aceite esencial que inhibe el crecimiento de $R$. solani. Diluciones del ensayo 1: 10, $100,1.000,10.000$ y $100.000 \mu \mathrm{L} \mathrm{mL}^{-1}$; ensayo 2 : 80, 85, 90, 95 y $100 \mu \mathrm{L} \mathrm{mL}^{-1}$ y ensayo 3: 100, 110, 120, 130 y $140 \mu \mathrm{L} \mathrm{mL}^{-1}$. En cada ensayo se utilizó un diseño experimental completamente al azar con quince repeticiones. La unidad experimental correspondió a una caja de Petri. Los tratamientos correspondieron a las concentraciones definidas en cada ensayo y comparadas con el testigo absoluto que no fue enmendado. 
Se midió el crecimiento micelial mediante Tpsdig2 cada 48 h hasta completar las 192 h. Se utilizó la temperatura $20^{\circ} \mathrm{C}$ para la incubación. El cálculo del porcentaje de inhibición $(P H)$ se describe como:

$$
P H=\frac{D C C-D C T}{D C C} \times 100
$$

donde DCC corresponde al diámetro de la colonia control, DCT diámetro de la colonia tratada (Nguyen et al., 2009; Álvarez et al., 2016). Los valores porcentuales se transformaron con la relación arcoseno $(\sqrt{ } P H / 100)$ y se evaluaron mediante análisis de varianza con prueba de comparación de medias de Tukey $(P=0,05)$.

Para el cálculo de la concentración efectiva cincuenta $\left(\mathrm{CE}_{50}\right)$, se utilizó un análisis de regresión lineal simple, tomando el porcentaje de inhibición $\mathrm{PH}$ como variable dependiente y los valores transformados con logaritmo natural de la concentración del aceite como variable independiente. Se calcularon los coeficientes de regresión, intercepción de la recta, coeficiente de determinación y el error estándar de la estimación, a partir del modelo se estimó la concentración que inhibe al $50 \%$.

Finalmente, después de un periodo de evaluación de 192 h se definió la concentración mínima inhibitoria (MCI) la cual fue la proporción más baja de aceite esencial que inhibió el crecimiento visible del fitopatógeno.

Análisis morfométrico $R$. solani. Con el fin de evaluar las posibles modificaciones en la forma del micelio de $R$. solani, se usó el valor $\mathrm{CE}_{50}$ determinado previamente para enmendar el medio de cultivo en comparación a un testigo sin aceite esencial. Los medios con el fitopatógeno se incubaron durante $192 \mathrm{~h}\left(20^{\circ} \mathrm{C}\right)$. Al cabo de este periodo, se montaron láminas de observación con azul de lactofenol como agente de tinción y seguimiento fotográfico al microscopio óptico (40X) para el registro.

Usando el programa informático ImageJ se estimó el contorno, diámetro y longitud de las hifas de $R$. solani. Con los puntos del contorno se realizó un análisis morfométrico con Series de Fourier Elípticas, tomando veinte mediciones por placa para generar el ajuste (Álvarez et al., 2016), a partir de los coeficientes del análisis elíptico se desarrolló un análisis multivariado para comparar el efecto del aceite esencial mediante el índice de Shapiro Wilk's (Álvarez et al., 2016; Medeiroset al., 2016).
Todos los análisis estadísticos se desarrollaron con ayuda del programa informático Statgraphics Centurion XVI.

Identificación de la fracción activa del aceite. Para el fraccionamiento del aceite esencial se utilizó la metodología de cromatografía en capa fina reportada por Moreira et al. (2011), con modificaciones propuestas para esta investigación. Se aplicó $0,75 \mu \mathrm{L}$ de aceite esencial de L. origanoides en una placa preparativa $[2,5 \times 6 \mathrm{~cm}$, con fase estacionaria sílica-gel (60G F254)], para su separación se utilizaron los solventes Tolueno:Acetato de etilo, en una relación 9:1 (v/v) como fase móvil. Se usó paralelamente a la inyección del aceite los estándares comerciales timol y carvacrol de la marca Sigma-Aldrich.

Las fracciones fueron identificadas mediante constantes $R f$ (Ratio of front), que expresan la posición de cada compuesto sobre la placa cromatográfica como la fracción decimal, a través de la ecuación:

$$
R f=\frac{L 1}{L 2}
$$

donde $L 1$ es la distancia recorrida por la muestra desde el punto de aplicación hasta el frente del solvente y L2 la distancia del frente del solvente (Nguyen et al., 2009).

La fracción activa del aceite esencial se determinó mediante bioautografía, para ello se usó el sistema cromatográfico descrito anteriormente, el cual fue secado por 10 min para completar la eliminación de los solventes. Seguidamente se dispuso la placa en la caja Petri y se cubrió con una capa fina de medio de cultivo para lo cual se empleó un spray de vidrio diseñado para esta investigación (Nguyen et al., 2009; Plodpai et al., 2013).

Para la inoculación de $R$. solani se ubicaron discos de agar de $1,1 \mathrm{~cm}$ de diámetro dispuestos a $20 \mathrm{~mm}$ de la placa cromatográfica y se incubó a $20^{\circ} \mathrm{C}$ por un periodo de 144 h, el montaje se repitió 20 veces.

La región donde el patógeno no se desarrolló fue atribuida a la fracción activa del aceite esencial. Esta fracción fue aislada de la placa cromatográfíca mediante raspado con espátula y lavado con diclorometano grado HPLC. La solución obtenida fue dispuesta en tubos Eppendorf sometidos a centrifugación $5.000 \mathrm{rpm}$, el sobrenadante se identificó mediante GC-MS. 


\section{RESULTADOS Y DISCUSIÓN}

\section{Perfil cromatográfico de aceite esencial}

Los rendimiento de extracción de aceite esencial alcanzaron $2,67 \pm 0,05 \%$. Se identificaron nueve compuestos con una proporción relativa superior a $1 \%$ los cuales representaron el 92,65\% del aceite (Tab. 1). Estos compuestos hacen parte del grupo de los terpenos, los cuales son producto de la biosíntesis del metabolismo secundario de L. origanoides, sintetizados a partir de la ruta del ácido mevalónico en el citosol de la célula, o mediante la ruta del metileritritol fosfato en los cloroplastos para dar origen a la unión de isopreno (compuestos 5 átomos de carbono) (Dewick, 2002).

El compuesto mayoritario del aceite esencial fue timol en $60,7 \%$, además se destacaron por su relativa abundancia $\rho$-cimeno, $\boldsymbol{\gamma}$-terpineno y mirceno (Tab. 1). Con referencia a las proporciones encontradas, fue posible clasificar la muestra de orégano silvestre procedente de la zona del Alto Patía, identificada con el código TEA-001 (N45202), como quimiotipo timol entre las tres clasificaciones reportadas para L. origanoides (Stashenko et al., 2010; Vicuña et al., 2010).

Tabla 1. Compuestos mayoritarios identificados en el aceite esencial de L. origanoides por GC-MS.

\begin{tabular}{|c|c|c|c|}
\hline No. & $\mathbb{R}$ & Identificación & Cantidad relativa (\%) \\
\hline 1 & 990 & Mirceno & 5,70 \\
\hline 2 & 1.015 & $\alpha$-Terpineno & 2,12 \\
\hline 3 & 1.024 & $\rho$-Cimeno & 8,85 \\
\hline 4 & 1.028 & Limoneno & 1,13 \\
\hline 5 & 1.058 & $\gamma$-Terpineno & 6,30 \\
\hline 6 & 1.304 & Timol & 60,7 \\
\hline 7 & 1.308 & Carvacrol & 0,15 \\
\hline 8 & 1.424 & $\beta$-Cariofileno & 5,13 \\
\hline 9 & 1.458 & $\alpha$-Humuleno & 2,70 \\
\hline
\end{tabular}

IR: índice de retención de Kovats en columna DB-5 (J\&W).

El timol se referencia en distintos estudios como parte mayoritaria del aceite esencial de orégano silvestre en proporciones que varían entre 29,6 y $66 \%$, atribuidas a las condiciones climáticas, altitud, época de cosecha, estado de crecimiento de la planta, así como el método y las condiciones de extracción del aceite esencial (Vicuña et al., 2010; Arango et al., 2012; Souza et al., 2013; Ospina et al., 2016).
Además, la proporción de timol encontrada se referencia como una de las mayores a nivel experimental, solamente superada por el ecotipo COL519799 colectado por Ramírez et al. (2009) en Bucaramanga (Santander, Colombia) con un porcentaje relativo del $66 \%$. Por tanto, se demuestra con los registros el potencial del orégano silvestre para la obtención de timol como compuesto secundario a nivel industrial, más aún cuando este arbusto es endémico de la ecoregión del Alto Patía.

\section{Actividad biocida del aceite esencial}

Se presentó sensibilidad de $R$. solani frente al aceite esencial de orégano silvestre a nivel in vitro; demostrado por el comportamiento en el porcentaje de inhibición $(\mathrm{PH})$ del fitopatógeno y la diferencia estadística estimada (Tab. 2).

\section{Tabla 2. Porcentaje de inhibición (PH) de $R$. solani frente al aceite esencial de $L$. origanoides a nivel in vitro.}

\begin{tabular}{|c|c|c|}
\hline Ensayo & $\begin{array}{c}\text { Concentración } \\
\left(\boldsymbol{\mu L} \mathrm{mL}^{-1}\right)\end{array}$ & $\begin{array}{c}\text { Porcentaje de inhibición } \\
\text { de } R \text {. solani }(\%)\end{array}$ \\
\hline \multirow{4}{*}{ Ensayo 1 } & 10 & $3,40 \mathrm{a}$ \\
\cline { 2 - 3 } & 100 & $82,8 \mathrm{e}$ \\
\cline { 2 - 3 } & 1.000 & $100 \mathrm{f}$ \\
\cline { 2 - 3 } & 10.000 & $100 \mathrm{f}$ \\
\hline \multirow{4}{*}{ Ensayo 2 } & 100.000 & $100 \mathrm{f}$ \\
\cline { 2 - 3 } & 80 & $24,16 \mathrm{~b}$ \\
\cline { 2 - 3 } & 85 & $49,30 \mathrm{c}$ \\
\cline { 2 - 3 } & 90 & $51,32 \mathrm{~cd}$ \\
\hline \multirow{4}{*}{ Ensayo 3 } & 95 & $59,57 \mathrm{~d}$ \\
\cline { 2 - 3 } & 100 & $81,00 \mathrm{e}$ \\
\cline { 2 - 3 } & 100 & $82,50 \mathrm{e}$ \\
\cline { 2 - 3 } & 110 & $100 \mathrm{f}$ \\
\cline { 2 - 3 } & 120 & $100 \mathrm{f}$ \\
\hline
\end{tabular}

Promedios con letras distintas indican diferencia significativa según la prueba de Tukey $(P \leq 0,05)$.

El resultado de los ensayos permitió calcular el modelo de regresión $y=453,37 x-830,63$ con coeficiente de determinación $R^{2}=0,976$ estableciendo la concentración $\mathrm{CE}_{50}$ en $88,94 \mu \mathrm{L} \mathrm{mL}^{-1}$. Por otro lado, al finalizar las $192 \mathrm{~h}$ el tercer ensayo se determinó que la concentración de aceite esencial $110 \mu \mathrm{L} \mathrm{mL}^{-1}$ fue causante de 
la inhibición de R. solani y por tanto se reporta como la concentración mínima inhibitoria (MCI).

Otros aceites esenciales también han sido evaluados sobre $R$. Solani sin encontrar los niveles de inhibición obtenidos con aceite de orégano silvestre. Por ejemplo, Khaledi et al. (2015) encontraron MCI de $850 \mu \mathrm{L}$ $\mathrm{mL}^{-1}$ con aceite de Mentha piperita L. y $1.200 \mu \mathrm{L} \mathrm{mL}^{-1}$ con aceite Bunium persicum B.Fedtsch. Igualmente, Seema y Devaki (2010) determinan MCI de $1.000 \mu \mathrm{L}$ $\mathrm{mL}^{-1}$ con el aceite de Syzygium aromaticum (L.) Merr. \& L.M.Perry.

Además, L. origanoides tiene reportes biocidas sobre microorganismos de interés agronómico como $P$. infestans (Arango et al., 2014; Álvarez et al., 2016), A. solani (Álvarez et al., 2016), B. cinerea (Taborda et al., 2015), Spongospora subterranea (Wallr.) Lagerh. (Bittara et al., 2009), Fusarium oxysporum Schltdl. (Tangarife et al., 2012) y Bipolaris maydis (Y. Nisik. \& C. Miyake) Shoemaker (Rodríguez y Sanabria, 2005), quienes reportan altos niveles de inhibición micelial en pruebas in vitro con reducción en la incidencia y severidad de la enfermedad in vivo. También estos autores coinciden en indicar la presencia de compuestos fenólicos y terpenos en los extractos etanólicos del aceite esencial de L. origanoides explicando la alta actividad biocida sobre los organismos probados.

\section{Análisis morfométrico de $R$. solani}

Se observaron cambios degenerativos en las hifas sometidas a la acción biocida del aceite de L. origanoides en comparación con el control, a lo cual Marei et al. (2012) indican que las características lipofílicas por contacto de los aceites esenciales le permiten entrar al citoplasma de los microorganismos y causar alteraciones morfológicas.
Se encontraron diferencia significativa $(P<0,05)$ en el diámetro de estructuras hifales entre los tratamientos. Se registraron diámetros de 3,12 $\mu \mathrm{m}$ en las hifas sometidas al aceite y $4,30 \mu \mathrm{m}$ en el testigo. Por el contrario, la longitud del cuerpo del hongo (hifa) para los tratamientos no presentaron diferencias estadísticas $(P=0,36)$, aun cuando la longitud de la hifa de $R$. solani sometida al aceite esencial presentó entre 4,47 y $5,50 \mu \mathrm{m}$ respecto al control que alcanzó $5,79 \mu \mathrm{m}$.

Se destaca a nivel microscópico que las hifas del hongo en el medio control presentaron un menor número de septos (Fig. 1A), contrario a lo observado en el tratamiento suplementado con aceite esencial en el cual se registraron hasta tres veces más divisiones de la estructura (Fig. 1B). No fue posible establecer en otra investigación un comportamiento similar en la morfología de $R$. solani, sin embargo, teniendo en cuenta la sensibilidad que mostró el microorganismo se sospecha que esto se deba a una respuesta de defensa.

Para el análisis de contorno, el índice de Shapiro Wilk's mostró diferencias altamente significativas $(P \leq 0,01)$ entre los tratamientos, con ello se puede concluir que el aceite reduce el área y vigor del cuerpo del microorganismo. Resultados similares fueron reportados por Nguyen et al. (2009), Amini et al. (2012) y Plodpai et al. (2013) al exponer $R$. solani a diferentes concentraciones de aceites esenciales, presentando cambios morfológicos en las hifas de este microorganismo, además de ruptura de la membrana plasmática y destrucción mitocondrial.

Los resultados encontrados en la afección sobre la morfología del fitopatógeno fue irreversible, a diferencia de los reportes de Nguyen et al. (2009), quienes indican que bajas concentraciones de productos botánicos evaluados pueden causar efectos reversibles sobre el microorganismo.

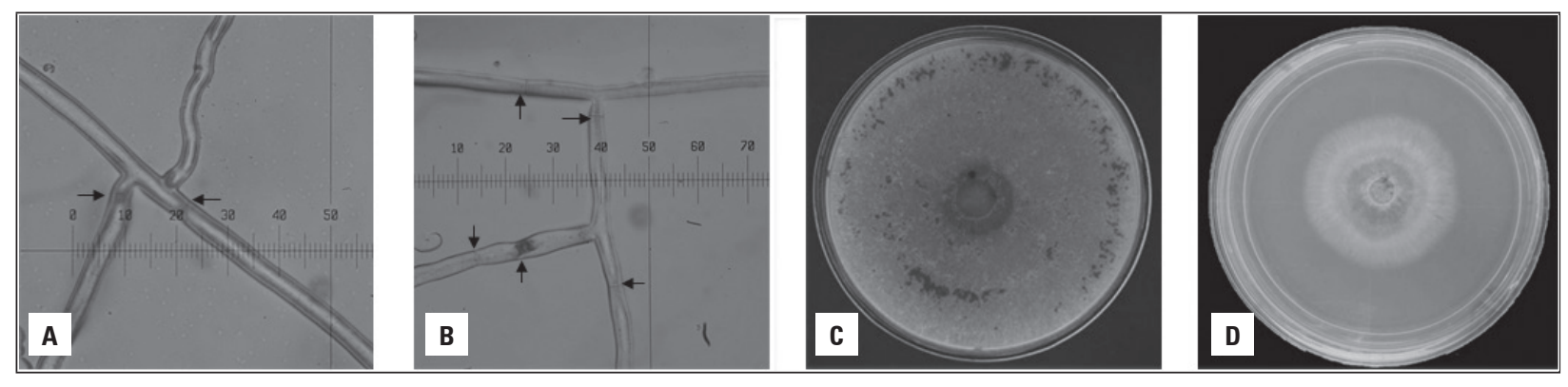

Figura 1. Diferencias macroscópica y microscópica de $R$. solani a las $192 \mathrm{~h}$ de crecimiento, medio de cultivo PDA suplementado con aceite esencial de $L$. origanoides. A y $C=$ tratamiento control; $B$ y $D=$ concentración $C_{50}\left(88,94 \mu \mathrm{LL}^{-1}\right)$. 


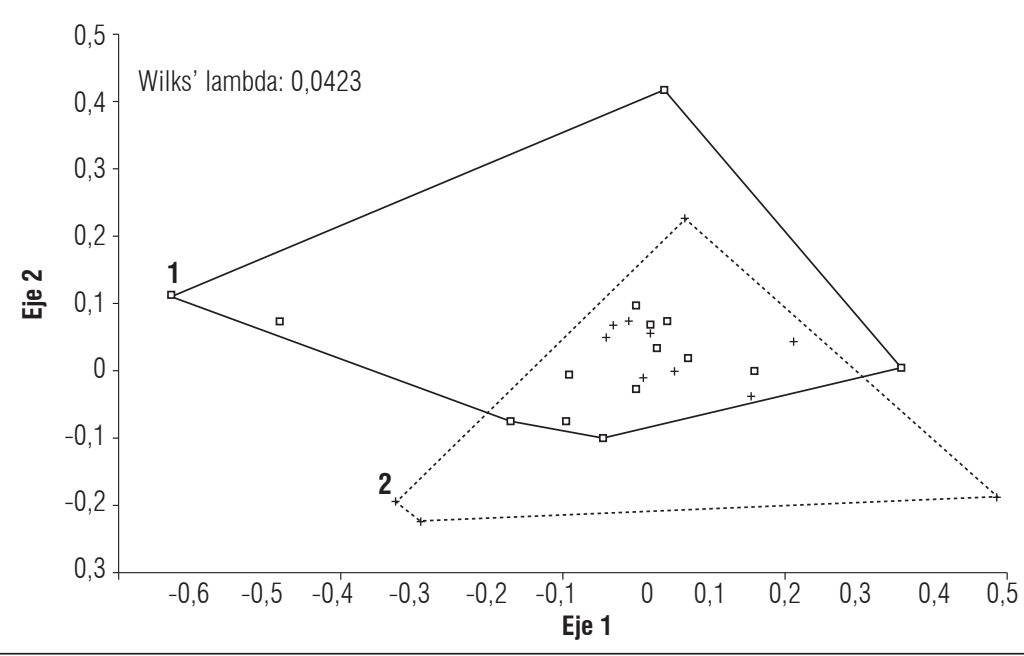

Figura 2. Análisis morfométrico del contorno de las hifas de $R$. solani sometidas al aceite esencial de $L$. origanoides. Tratamiento control (1) y tratamiento con aceite (2).

A nivel macroscópico la inhibición en el crecimiento de $R$. solani, también presentó reducción en el volumen micelial y ausencia de esclerocios respecto al control (Fig. 1C). Se ha sugerido que los aceites esenciales interfieren especialmente sobre la producción enzimática de los microorganismos, afectando los procesos constitutivos y metabólicos. Para el caso de R. solani, Abd-El-Khair y El-Gamal (2011) proponen que la capacidad de colonización y reproducción del fitopatógeno se ve afectada por la disminución de celulasas y pectinasas.

\section{Fracción activa del aceite esencial de L. origanoides}

La separación del aceite esencial de L. origanoides en los folios cromatográficos permitió la visualización de cuatro fracciones identificables mediante luz ultra violeta $(\lambda=254 \mathrm{~nm})$ con valores de relación de frentes $R_{f}$ para la fracción 1 de 0,14; fracción 2 de 0,33; fracción 3 de 0,60 y fracción 4 de 0,74 (Fig. 3A).

Después de inocular el microorganismo sobre la placa cromatográfica se evidenció la restrinción del desarrollo en la tercera fracción $\left(R_{f}=0,60\right)$, lo cual se le atribuye a la acción biocida mediante esta técnica (Fig. 3B). Una vez determinado esto, se procedió a identificar la fracción a través de GC-MS y la comparación del recorrido con el patrón químico, dando como resultado que el compuesto detectado fue timol.

El mecanismo de acción del timol en la actualidad no se encuentra dilucidado totalmente, sin embargo

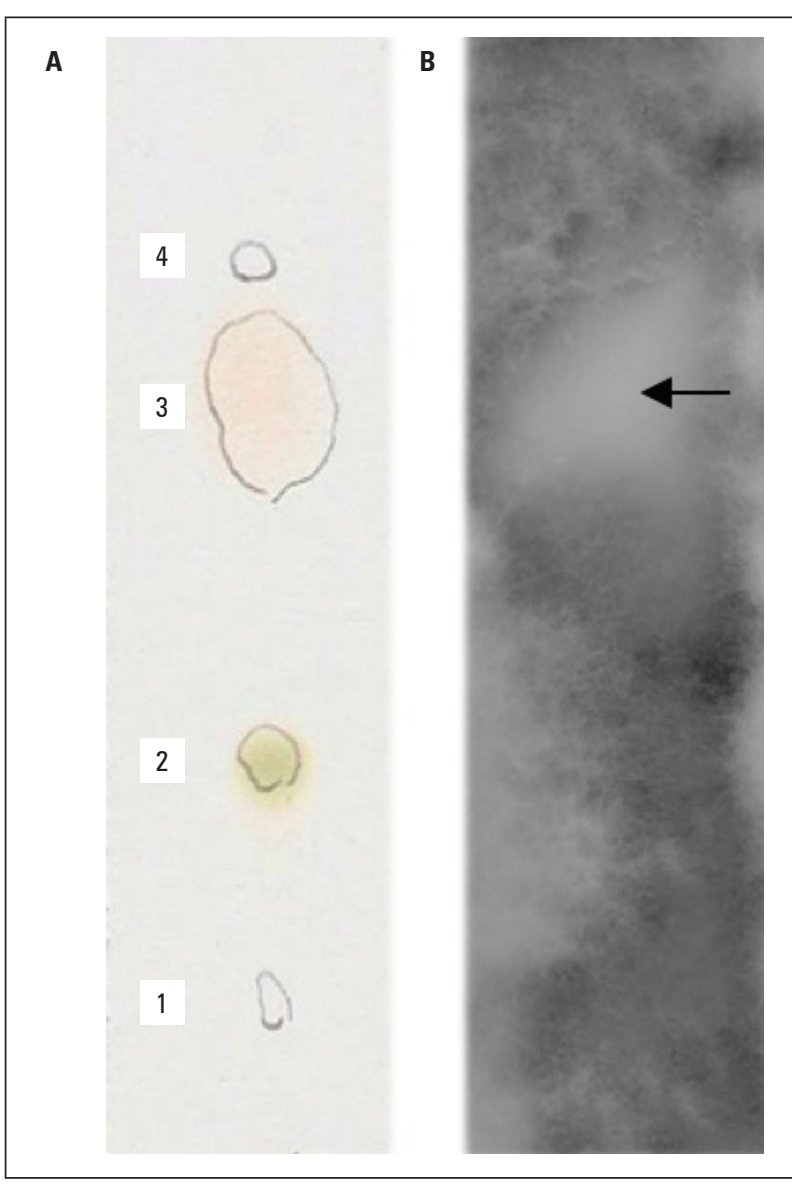

Figura. 3. Fracción activa del aceite esencial de $L$. origanoides. (A) fraccionamiento del aceite esencial mediante Cromatografía en Capa Fina, (B) fracción activa para $R$. solani. 
autores como Marei et al. (2012) y Souza et al. (2013) han indicado que este afecta el intercambio de compuestos en la membrana citoplasmática de los microorganismos, lo cual interfiere en procesos de transducción de señales que afectan la función de algunas proteínas a nivel sistémico. Los resultados encontrados por los anteriores autores también indican que el timol causa cambios en la estructura y adhesión celular, plasticidad del cuerpo y modificación del $\mathrm{pH}$ que originó un cambio en el contenido iónico de la estructura.

Los daños ocasionados del timol a estos microorganismos está dirigido al citoplasma provocando cambios en la organización general y pérdida de la integridad del sistema estructural (membranas), lo que contribuye a la muerte de los blancos biológicos (Hoyos et al., 2012), como se evidenció en esta investigación.

Con la información obtenida es posible proyectar el desarrollo de un bioinsumo que pueda integrarse en los planes de manejo fitosanitario del cultivo de papa, otorgando una nueva estrategia que reduzca el uso de pesticidas sintéticos en la agricultura.

\section{CONCLUSIONES}

El aceite esencial de Lippia origanoides en condiciones in vitro presentó actividad biocida sobre Rhizoctonia solani, la concentración $110 \mu \mathrm{L} \cdot \mathrm{mL}^{-1}$ causó la inhibición en el desarrollo del fitopatógeno evidenciando el potencial como agente antifúngico natural.

La técnica de bioautografia permitió determinar que el timol, componente mayoritario del aceite esencial de L. origanoides $(60,7 \%)$ confiere las características biocidas.

El análisis morfométrico determinó que existen alteraciones en la estructura micelial de $R$. solani a causa del aceite esencial de L. origanoides disminuyendo el diámetro micelial y el área de contorno.

Conflicto de intereses: el manuscrito fue preparado y revisado con la participación de los autores, quienes declaran no tener algún conflicto de interés que coloque en riesgo la validez de los resultados aquí presentados.

\section{REFERENCIAS BIBLIOGRÁFICAS}

Abd-El-Khair, H. y N. El-Gamal. 2011. Effects of aqueous extracts of some plant species against Fusarium solani and Rhizoctonia solani in Phaseolus vulgaris plants. Arch. Phytopathol. Plant Prot. 44(1), 1-16. Doi: 10.1080/03235400802678436

Álvarez, D., A. Hurtado y J. Acosta. 2016. Aceite esencial de Lippia origanoides como posible producto biorracional en el cultivo de papa (Solanum tuberosum). Vitae 23(1), 526-530.

Amini, M., N. Safaie, M. Salmano y M. Shams. 2012. Antifungal activity of three medicinal plant essential oils against some phytopathogenic fungi. Trakia J. Sci. 10(1), 1-8.

Arango, O., F. Bolaños, O. Villota, A. Hurtado y I. Toro. 2012. Optimization of yield and thymol content of wild oregano essential oil obtained by steam distillation process. Biotecnol. Sect. Agropecu. Agroind. $10(2), 217-226$.

Arango, O., A. Hurtado, D. Pantoja y L. Santacruz. 2014. Actividad inhibitoria del aceite esencial de Lippia origanoides H.B.K sobre el crecimiento de Phytophthora infestans. Acta Agron. 64(2), 116-124. Doi: 10.15446/ acag.v64n2.42964

Barbosa, F., L. Barbosa, E. Melo, F. Botelho y R. Santos. 2006. Influência da temperatura do ar de secagem sobre o teor e a composição química do óleo essencial de Lippia alba (Mill) N. E. Brown. Química Nova 29(6), 1221-1225. Doi: 10.1590/S0100-40422006000600014

Bittara, F., D. Rodríguez, M. Sanabria, J. Monroy y J. Rodríguez. 2009. Evaluación de fungicidas y productos vegetales en el combate de la sarna polvorienta de la papa. Interciencia 34(4), 265-269.

Dewick, P. 2002. The biosynthesis of C5-C25 terpenoid compounds. Natural Prod. Rep. 19(1), 181-222. Doi: 10.1039/b002685i

Hoyos, J., E. Alves, L. Rozwalka, E. Souza y W. Zeviani. 2012. Antifungal activity and ultrastructural alterations in Pseudocercospora griseola treated with essential oils. Ciênc. Agrotecnol. 36(3), 270-284. Doi: 10.1590/ S1413-70542012000300002

Kankam, F., H. Qiu, L. Pu, H. Long, C. Zhang, J. He y H. Zhang. 2016. Isolation, purification and characterization of phytotoxins Produced by Rhizoctonia solani AG3 , the cause agent of potato stem canker. Am. J. Potato Res. 93(4), 321-330. Doi: 10.1007/s12230-016-9506-8

Khaledi, N., P. Taheri y S. Tarighi. 2015. Antifungal activity of various essential oils against Rhizoctonia solani and Macrophomina phaseolina as major bean pathogens. J. Appl. Microbiol. 118(3), 704-717. Doi: 10.1111/ jam.12730 
Marei, G., M. Abdel y S. Abdelgaleil. 2012. Comparative antifungal activities and biochemical effects of monoterpenes on plant pathogenic fungi. Pestic. Biochem. Physiol. 103(1), 56-61. Doi: 10.1016/j. pestbp.2012.03.004

Medeiros, F., F. Gouveia, H. Bizzo, R. Vieira y C. Del Menezzi. 2016. Fungicidal activity of essential oils from Brazilian Cerrado species against wood decay fungi. Int. Biodeterior. Biodegradation 114(2016), 87-93. Doi: 10.1016/j.ibiod.2016.06.003

Medeiros, H., I. de Lima, K. Nunes, L. Osorio, R. de Almeida, B. dos Santos, H. Melo, A. Lira, M. Freire, A. Lopes y J. Dantas. 2014. Effect of Lippia origanoides H.B.K. essential oil in the resistance to aminoglycosides in methicillin resistant Staphylococcus aureus. Eur. J. Integr. Med. 6(5), 560-564. Doi: 10.1016/j.eujim.2014.03.011

Moreira, M., G. Newandram, O. Dev, C. Manrangon, R. Coura y V. Moreira. 2011. Composition, antifungal activity and main fungitoxic components of the essential oil of Mentha piperita L. J. Food Saf. 32(1), 2936. Doi: 10.1111/j.1745-4565.2011.00341.x

Nguyen, V., D. Nguyen, D. Seo, R. Park y W. Jung. 2009. Antimycotic activities of Cinnamon-derived compounds against Rhizoctonia solani in vitro. BioControl 54(5), 697-707. Doi: 10.1007/s10526-009-9220-2

Ospina, J., C. Grande, J. Menjivar y M. Sanchez. 2016. Relación entre índice de refracción y la concentración de Timol en aceites esenciales de Lippia origanoides Kunth. Chil. J. Agric. Anim. Sci. ex Agro-Ciencia 32(2), 127-133.

Plodpai, P., S. Chuenchitt, V. Petcharat, S. Chakthong y S. Voravuthikunchai. 2013. Anti-Rhizoctonia solani activity by Desmos chinensis extracts and its mechanism of action. Crop Prot. 43(1), 65-71. Doi: 10.1016/j. cropro.2012.09.004

Ramírez, L., J. Isaza, L. Veloza, E. Stashenko y D. Marín. 2009. Actividad antibacteriana de aceites esenciales de Lippia origanoides de diferentes orígenes de Colombia. Ciencia 17(4), 313-321.
Rodríguez, D. y M. Sanabria. 2005. Efecto del extracto de tres plantas silvestres sobre la Rizoctoniasis, la mancha sureña del maíz y los patógenos que las causan. Interciencia 30(12), 739-744.

Seema, M. y N. Devaki. 2010. Effect of some essential oils on Rhizoctonia solani Kuhn infecting flue-cured virginia tobacco. J. Biopestic. 3(3), 563-566.

Souza, E., C. Oliveira, T. Stamford, M. Conceição y N. Gomes. 2013. Influence of carvacrol and thymol on the physiological attributes, enterotoxin production and surface characteristics of Staphylococcus aureus strains isolated from foods. Braz. J. Microbiol. 44(1), 29-35. Doi: 10.1590/S1517-83822013005000001

Stashenko, E., J. Martínez, C. Ruíz, G. Arias, C. Durán, W. Salgar y M. Cala. 2010. Lippia origanoides chemotype differentiation based on essential oil GC-MS and principal component analysis. J. Sep. Sci. 33(1), 93-103. Doi: $10.1002 /$ jssc. 200900452

Taborda, L., M. Sánchez, R. Bonilla y C. Huertas. 2015. Efecto fungistático de extractos y aceites esenciales de Lippia origanoides HBK y Thymus vulgaris L. como alternativas de manejo de Botrytis cinerea en fresa. Acta Agron. 64(1), 93-99. Doi: 10.15446/acag.v64n1.35773

Tangarife, V., V. Roa, L. Betancur, D. Durán, E. Stashenko y A. Mesa. 2012. Antifungal activity of Verbenaceae ancl Labiatae families essential oils. Pharmacology online 1(1), 43-55.

Vicuña, G., E. Stashenko y J. Fuentes. 2010. Chemical composition of the Lippia origanoides essential oils and their antigenotoxicity against bleomycin-induced DNA damage. Fitoterapia 81(5), 343-349. Doi: 10.1016/j.fitote.2009.10.008

Villa, A., R. Pérez, H. Morales, M. Basurto, J. Soto y E. Martínez. 2015. Situación actual en el control de Fusarium spp. y evaluación de la actividad antifúngica de extractos vegetales. Acta Agron. 64(2), 194-205. Doi: 10.15446/acag.v64n2.43358 